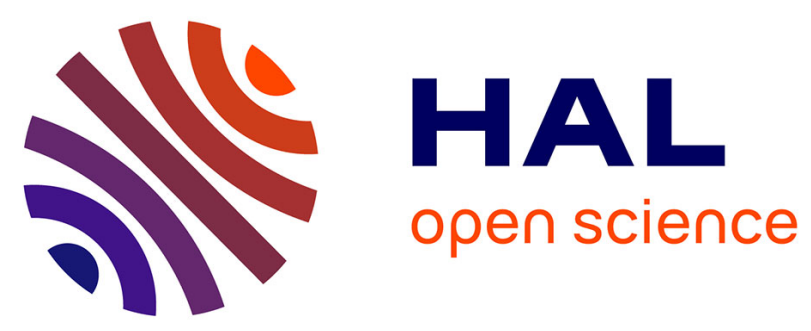

\title{
Immunity against Mycobacterium ulcerans - The subversive role of mycolactone
}

Caroline Demangel

\section{To cite this version:}

Caroline Demangel. Immunity against Mycobacterium ulcerans - The subversive role of mycolactone. Immunological Reviews, 2021, 301 (1), pp.209-221. 10.1111/imr.12956 . pasteur-03246855

\section{HAL Id: pasteur-03246855}

https://hal-pasteur.archives-ouvertes.fr/pasteur-03246855

Submitted on 2 Jun 2021

HAL is a multi-disciplinary open access archive for the deposit and dissemination of scientific research documents, whether they are published or not. The documents may come from teaching and research institutions in France or abroad, or from public or private research centers.
L'archive ouverte pluridisciplinaire HAL, est destinée au dépôt et à la diffusion de documents scientifiques de niveau recherche, publiés ou non, émanant des établissements d'enseignement et de recherche français ou étrangers, des laboratoires publics ou privés.

\section{()ㅜ) $\Theta$}

Distributed under a Creative Commons Attribution - NoDerivatives| 4.0 International 
Immunity against Mycobacterium ulcerans - the subversive role of

\section{mycolactone}

Caroline Demangel*

Immunobiology of Infection Unit, Institut Pasteur, INSERM U1221, Paris, France

* Correspondence: Caroline Demangel, Institut Pasteur, 25 Rue du Dr Roux, 75724 Paris

Cedex 15, France. Phone: 33 (0)1 406130 66; e-mail: demangel@pasteur.fr 


\section{Summary}

Mycobacterium ulcerans causes Buruli ulcer, a neglected tropical skin disease manifesting as chronic wounds that can leave victims with major, life-long deformity and disability. Differently from other mycobacterial pathogens, M. ulcerans produces mycolactone, a diffusible lipid factor with unique cytotoxic and immunomodulatory properties. Both traits result from mycolactone targeting Sec61, the entry point of the secretory pathway in eukaryotic cells. By inhibiting Sec61, mycolactone prevents the host cell's production of secreted proteins, and most of its transmembrane proteins. This molecular blockade dramatically alters the functions of immune cells, thereby the generation of protective immunity. Moreover, sustained inhibition of Sec61 triggers proteotoxic stress responses leading to apoptotic cell death, which can stimulate vigorous immune responses. The dynamics of bacterial production of mycolactone and elimination by infected hosts thus critically determine the balance between its immuno-stimulatory and -suppressive effects. Following an introduction summarizing the essential information on Buruli ulcer disease, this review focuses on the current state of knowledge regarding mycolactone's regulation and biodistribution. We then detail the consequences of mycolactone-mediated Sec61 blockade on initiation and maintenance of innate and adaptive immune responses. Finally, we discuss the key questions to address in order to improve immunity to M. ulcerans, and how increased knowledge of mycolactone biology may pave the way to innovative therapeutics. 


\section{I - Introduction - Key facts about Buruli ulcer disease}

\section{I.1. Epidemiology}

Buruli ulcer (BU) is a neglected tropical disease caused by Mycobacterium ulcerans, which manifests as chronic, nonhealing necrotic skin lesions. Between 1960 and 2015, BU was reported in 34 countries primarily located in tropical and subtropical areas ${ }^{1,2}$. In these countries, BU periodically emerges as small outbreaks in geographically limited foci ${ }^{3}$. West African countries are the worst affected, with prevalence estimates reaching 26.9 cases per 10,000 population in Benin ${ }^{1,4}$. Available epidemiological data likely underestimate the real burden and distribution of $\mathrm{BU}$, due to under-reporting and misdiagnosis of the disease ${ }^{4}$. In 1998, the rising incidence of BU in Western Africa prompted policy makers to adopt the Yamoussoukro Declaration, expressing commitment to intensify actions against BU. Since then, the WHO Global BU Initiative has coordinated active surveillance and control programs, as well as research efforts, to stop BU disease expansion. In 2013, programmatic targets were defined to ensure reliable PCR diagnosis, promote early case finding and reduce the sequelae and disability that are associated with severe BU. Although effective at reducing the global incidence of BU until 2017, these measures failed to eradicate BU ${ }^{5}$. Today, most of the program objectives formulated by the WHO remain unmet and local epidemics keep arising ${ }^{5}$. It is therefore important to revitalize global control and research efforts to prevent the reemergence of $\mathrm{BU}{ }^{5}$.

\section{I.2. Clinical presentation - Diagnosis}

BU may start as a swelling nodule, a large area of induration or as a diffuse edema ${ }^{6}$. Within weeks to months, these lesions develop into open ulcers with a characteristic lack of pain and 
inflammation, which if untreated enlarge over time ${ }^{7}$. BU ulcers are categorized by three levels of increasing severity depending on the number and importance of skin lesions and bone involvement ${ }^{7}$. Well trained, experienced health professionals usually make a reliable clinical diagnosis without laboratory confirmation ${ }^{8}$. However, early nodules and ulcerative BU lesions may be confused with other conditions such as diabetic ulcers, cutaneous leishmaniasis or yaws, and specific tests can be necessary to confirm BU diagnosis. Several methods are available to diagnose BU: molecular detection of $M$. ulcerans-specific IS2404 insertion sequence, direct microscopy, histopathology and culture ${ }^{9}$. While sensitive and highly specific 10,11, BU diagnostic tests based on bacterial DNA detection by polymerase chain reaction (PCR) or loop-mediated isothermal amplification (LAMP) techniques ${ }^{12}$ require trained laboratory staff and infrastructure that are often missing in field conditions ${ }^{5}$.

\section{I.3. Treatment}

The WHO currently recommends an 8-week long daily administration of oral rifampicin plus intramuscular streptomycin, associated with wound management and surgery for severe lesions ${ }^{13}$. While rare, mutations associated with drug resistance do occur ${ }^{14}$ and require careful monitoring. Recently, Phillips et al. reported that BU is curable by an all-oral, 8-week course of fully oral rifampicin plus clarithromycin with minimum use of surgery ${ }^{15,16}$. From an immunological perspective, it is interesting to note that patients undergoing antibiotic treatment report considerable pain. Moreover, paradoxical worsening of the lesions, with enlargement of the treated ulcer and sometimes development of secondary lesions, is often observed after the start of antimicrobial treatment ${ }^{17-22}$. Such reactions may be interpreted as a treatment failure. However, histopathological and clinical examination of involved tissues argue that on the contrary, they result from immune-mediated reactions to effective, 
microbiologically sterilizing treatments (see $§$ III). Practically, this means that BUs are generally not healed at the end of antibiotic therapy, and patients require close follow-up with adequate wound management until completely cured.

\section{I.4. Evolution - Transmission}

Bacterial population genomics revealed that $M$. ulcerans evolved from a $M$. marinum ancestor and disseminated across the world and particularly within Africa, the continent most affected by $\mathrm{BU}$ today ${ }^{23}$. During its evolution, $M$. ulcerans underwent considerable genome reduction 24-27 while acquiring the unique ability to make mycolactone ${ }^{6,24,28}$. M. ulcerans is an environmental bacterium, whose mode of transmission to humans remains largely unknown. M. ulcerans bacilli grow optimally at temperatures between $30-33^{\circ} \mathrm{C}$ and their growth is inhibited at higher temperatures ${ }^{29,30}$. This limited growth temperature range is believed to explain why the pathogen does not disseminate beyond cutaneous and subcutaneous tissues in infected hosts. However, relative skin temperature is not a major determinant of BU body distribution. The topography of lesions in BU patients rather suggests a complex association of contributing factors such as pre-existing skin injury or exposure to biting insects ${ }^{31}$.

Epidemiologic and genetic analyses of family clusters of BU patients argue against human-tohuman transmission ${ }^{32}$. Living or working near stagnant waters was identified as a risk factor for M. ulcerans infection ${ }^{33}$, and there is growing evidence to suggest that natural or humandriven perturbations in landscape hydrology that create lentic habitats promote the emergence of new BU foci ${ }^{34,35}$. Based on PCR detection of $M$. ulcerans DNA in environmental samples (reviewed in ${ }^{36}$ ) and experimental infection of animal models ${ }^{37,38}$, the current view is that skin gets infected by a combination of contamination and puncture, including by insect 
bites $^{36}$. In addition to human beings, $M$. ulcerans infects a broad range of animals constituting potential environmental reservoirs ${ }^{29,39-41}$, and transmission pathways may depend on local ecosystems ${ }^{29,35,36}$.

In all, BU is a re-merging neglected tropical disease deserving renewed attention. Despite a major breakthrough in definition and validation of antibiotic therapy, there remain significant research gaps including the elucidation of BU transmission mechanisms, reasons for the timelag between microbiological and clinical cure and identification of prevention measures. Much of this knowledge resides in a better understanding of mycolactone biology. The next paragraphs review the state of art in genetic basis of mycolactone synthesis, regulation, biodistribution in organisms infected with $M$. ulcerans, as well as molecular target and mechanism of action.

\section{II - Mycolactone: bacterial synthesis and distribution in infected hosts}

\section{1. Genetic basis of mycolactone production and regulation}

Mycolactone was first described in 1999 by Pamela Small and col. in a seminal paper reporting the isolation of a polyketide-derived macrolide from a clinical isolate of $M$. ulcerans, which caused cytopathicity in cultured fibroblasts ${ }^{42}$. Subtractive hybridization between strains of $M$. ulcerans and M. marinum subsequently led to the identification of a polyketide synthase (PKS) locus that is specific to M. ulcerans ${ }^{43}$, then to the discovery of a $174-\mathrm{kb}$ plasmid (pMUM) encoding the giant PKSs synthesizing mycolactone ${ }^{44}$. Since then, other mycolactone- 
producing mycobacteria (MPMs) have been isolated from fish, frogs and other ectotherms presenting BU-like diseases (reviewed in ${ }^{23}$ ). Mycolactones produced by M. ulcerans strains of different geographical origins or genetically related mycobacteria are all variants of a canonical structure, corresponding to a 12-membered lactone ring substituted with two polyketide-derived chains ${ }^{24,45}$. While initially given new species names, MPMs were found to share extensive genomic homology with $M$. ulcerans ${ }^{25,46}$. They all harbor pMUM-like plasmids, produce mycolactone variants and are now considered different members of a single $M$. ulcerans species ${ }^{25,28,45-47}$. Notably, clinical isolates of $M$. ulcerans always produce mycolactone, indicating that maintenance of the pMUM plasmid is essential for in vivo persistence ${ }^{24,48}$. Mycolactone may also promote bacterial survival outside of human hosts, by promoting the formation of biofilms and/or the colonization of insect vectors ${ }^{49}$.

Whether and how mycolactone production is regulated is largely unknown. The PKS locus occupies a large part of the pMUM megaplasmid and involves the $\mathrm{m} / \mathrm{s} A 1, \mathrm{~m} / \mathrm{s} A 2$ and $\mathrm{m} / \mathrm{s} B$ genes, whose expression is driven by a strong SigA-like promoter sequence ${ }^{50}$. By experimentally infecting mice with a $M$. ulcerans strain expressing a GFP-reporter plasmid under the control of this promoter, Tobias et al. found that genes encoding mycolactoneproducing PKSs were highly expressed by bacilli present in ulcerated tissues ${ }^{50}$. This suggested that bacteria actively produce mycolactone in vivo. In line with these findings, mycolactone was detected in pre-ulcerative lesions, edges and exudates of ulcers from patients with active BU ${ }^{51,52}$. In M. ulcerans grown in vitro, toxin synthesis was decreased by the presence of carbohydrates despite elevated expression of $\mathrm{m} / \mathrm{s}$ genes, indicating that bacterial production of mycolactone may be modulated by environmental signals via post-transcriptional mechanisms ${ }^{53}$. 


\section{2. Secretion and biodistribution}

The molecular mechanisms mediating mycolactone export by $M$. ulcerans bacilli are not yet fully understood. Mycolactone was initially isolated from bacterial culture supernatants ${ }^{42}$, indicating that the toxin is secreted by the bacteria. Subsequent studies identified mycolactone in an extracellular matrix enveloping clusters of M. ulcerans bacilli, which was proposed to constitute a toxin reservoir ${ }^{49}$. While the cell wall localization of PKSs suggested that mycolactone biosynthesis occurs there, the toxin's assembly appears to require additional, unknown elements ${ }^{54}$.

Despite the lack of molecular tools and techniques for sensitive in-situ detection of mycolactone, studies using radio- or fluorescently-labelled molecule and analyses of organic solvent-extracted lipids have generated insights into how mycolactone distributes in infected hosts, at both cellular and tissue levels ${ }^{6}$. The next paragraphs provide an overview of recent advances in the field, with a particular focus on cells and organs of the immune system.

\section{II.2.a. Entering and leaving the bloodstream}

Mycolactone being lipophilic, its presence in biological samples was first assessed by extraction of total lipids with organic solvents, followed by analysis by liquid chromatography tandem-mass spectrometry (LC-MS/MS). Using this approach, structurally-intact mycolactone was detected in serum samples from newly diagnosed BU patients ${ }^{52}$, providing the first evidence that mycolactone produced by bacteria infecting the skin gains access to the bloodstream. However, the poor sensitivity of this approach prevented accurate determinations of the low concentrations of circulating mycolactone. Recent investigations 
indicated that mycolactone associates with human High- and Low-Density Lipoproteins (HDL and $\mathrm{LDL}$ ) in vitro ${ }^{55}$. This property may be used to capture, concentrate and quantitate mycolactone in patient serum.

The distinctive mass spectrometric signature of the toxin was also detected in lipid extracts of peripheral blood cells, spleen, liver and kidneys from mice experimentally infected with $M$. ulcerans ${ }^{56}$. When mice were injected with a radiolabeled form of the toxin via the subcutaneous, intravenous or intraperitoneal routes, radioactivity was detected in peripheral blood, spleen, liver and kidneys, further showing the body-wide distribution of mycolactone ${ }^{56}$. Whether injected or produced by bacteria, mycolactone was detected in the mononuclear cell fraction of blood, lymph nodes and spleen ${ }^{56}$. Further, when incubated with whole blood or spleen cell suspensions ex vivo, mycolactone rapidly distributed in the mononuclear cell compartment ${ }^{56}$. This indicated that in infected organisms, immune cells of blood and lymphoid organs are exposed to bacterially-produced mycolactone.

Recently, Guenin-Macé et al. used a fluorescently-labeled mycolactone and the zebrafish model to visualize in real-time the extravascular distribution of intravenously-delivered toxin 57. In agreement with mouse studies, injected fish displayed a body-wide distribution of mycolactone. Interestingly, the authors reported a concentration gradient around the site of injection that is reminiscent of the pathology of BU lesions, where tissue necrosis is centered on bacterial foci ${ }^{58}$. Notably, they observed a low-level persistence of mycolactone in the bloodstream of injected fish, despite important toxin clearance via the gastro-intestinal tract 57. In patients with $\mathrm{BU}$, the presence of mycolactone was still detectable in perilesional skin weeks after completion of antibiotic therapy ${ }^{51,52}$. Together, data from animal models and BU 
patients thus support the view that bacterially-produced mycolactone has a large distribution volume and a slow elimination rate, a pharmacokinetic profile that is compatible with high tissue binding capacity and relatively minor association to carrier proteins in serum. Albeit low, serum levels of mycolactone may constitute good indicators of its persistence in the organism, of major interest for clinical monitoring of disease.

\section{II.2.b. Crossing the plasma membrane}

Studies using fluorescent derivatives of mycolactone indicated that the toxin primarily penetrates host cells by passive diffusion ${ }^{59}$. Since mycolactone bound to HDL/LDL in vitro, it will be interesting to determine if lipid carrier protein receptors contribute, at least partially, to its intracellular uptake. Importantly, recent investigations using phospholipid membrane models indicated that mycolactone alters the integrity of membrane lipids ${ }^{60,61}$. Mycolactonephospholipid interactions were promoted by the presence of cholesterol and resulted in significant changes in lipid layer physical properties and architecture, including the formation

of ordered microdomains ${ }^{60,61}$. We can speculate that in immune cells, mycolactone insertion in cholesterol-rich lipid-rafts will affect the assembly of signaling platforms and associated immune responses. In support of this hypothesis, we observed that mycolactone promotes recruitment to lipid rafts and constitutive activation of the Src family kinase Lck in T lymphocytes ${ }^{62}$.

In sum, current data indicate that bacterially-produced mycolactone diffuses into the systemic circulation and gains access to distant organs (Figure 1). In the blood, mycolactone distributes primarily into leukocytes, which transport the toxin to secondary lymphoid organs. Mycolactone may also exist freely in peripheral blood or bound to lipoproteins, potentially 
delivering mycolactone to peripheral tissues via dedicated receptors. At the cellular level, mycolactone crosses the plasma membrane to reach the cytosol via passive diffusion, leading to structural perturbations in lipid bilayers.

\section{III - Mycolactone impact on inflammation and innate immunity}

Upon infection, professional phagocytic cells such as neutrophils and macrophages are normally recruited to the infection site to release cytokines and chemokines that promote inflammation and ensure efficient clearance of bacteria and apoptotic cells. Despite the presence of abundant bacilli and extensive tissue damage, BU lesions are characterized by a relative lack of inflammatory infiltrates and pain ${ }^{6}$. Experiments using intradermally-injected mice showed that $M$. ulcerans bacilli are initially captured by host phagocytes and transported to draining lymph nodes similarly to $M$. bovis BCG, leading to the initiation of vigorous Th-1oriented cellular immune responses ${ }^{63}$. Mycolactone-producing, but not mycolactonedeficient strains of $M$. ulcerans then induced the formation of expanding necrotic acellular foci ${ }^{64}$, suggesting that mycolactone produced by internalized bacteria kill host phagocytes. At late stages of mouse infection, M. ulcerans was primarily found as clusters of extracellular bacteria within localized area of necrosis ${ }^{63-65}$. The presence of leukocytes in an infiltration belt surrounding BU lesions, but not within the acellular necrotic core harboring extracellular bacteria, led Ruf et al. to propose that clusters of $M$. ulcerans bacilli reaching a critical size generate a mycolactone shield protecting the pathogen from host phagocytes ${ }^{66}$. 
Whether mycolactone impairs neutrophil sensing of pro-inflammatory mediators and migration towards the site of infection was not precisely investigated, but may operate since most of the membrane receptors mediating these biological processes (G protein-coupled, Fc, adhesion, cytokine and pattern recognition receptors) ${ }^{67}$ are predicted targets of mycolactone-mediated Sec61 blockade ${ }^{68}$ (see $\S \mathrm{V}$ ). In mice, mycolactone-producing and deficient strains of $M$. ulcerans both induced an acute neutrophilic response ${ }^{64}$. Intact neutrophils were detected in the infiltration belt surrounding established lesions of BU patients and neutrophilic debris were observed in their necrotic core, suggesting an early neutrophil infiltration ${ }^{66}$. Human blood-derived neutrophils pre-treated with non-cytotoxic doses of mycolactone ex vivo lost ability to produce pro-inflammatory TNF- $\alpha$ cytokine upon activation ${ }^{69}$. While insufficient to fully prevent neutrophil influx at early stages of infection, bacterial production of mycolactone may thus limit the capacity of infiltrated neutrophils to orchestrate and regulate inflammatory responses via cytokines and chemokines ${ }^{70}$.

Intracellular bacilli were detected within macrophages in the inflammatory infiltrates surrounding lesions of BU patients and experimentally infected mice ${ }^{64,65}$, demonstrating that M. ulcerans is transiently intracellular in macrophages of infected hosts. Torrado et al. showed that M. ulcerans may even multiply inside macrophages before inducing host cell lysis ${ }^{65}$. The ability of host macrophages to kill intracellular M. ulcerans before they are killed by bacterial production of mycolactone may be critical for the outcome of infection ${ }^{71}$. Expression of inducible nitric oxide synthase (iNOS) in macrophages stimulated by T cell-derived IFN- $\gamma$ is essential for control of $M$. tuberculosis infection ${ }^{72}$. Mice lacking IFN- $\gamma$ had reduced capacity to control M. ulcerans growth at early stages of infection ${ }^{73}$, suggesting that the IFN- $\gamma /$ iNOS signaling axis is also important in this disease context. Mycolactone efficiently decreased the 
surface expression of IFN- $\gamma$ receptor by macrophages, thereby compromising their ability to produce iNOS in response to IFN- $\gamma$ stimulation ${ }^{74}$. In accordance with these data, exogenouslyadded mycolactone dose-dependently inhibited phagosome maturation and nitric oxide production in IFN- $\gamma$-activated macrophages ${ }^{75}$. Together, these data strongly suggest that mycolactone-mediated suppression of IFN- $\gamma$ signaling in host macrophages undermines host innate immunity to $M$. ulcerans infection ${ }^{71}$. In support of this hypothesis, single nucleotide polymorphisms reducing iNOS and IFNG gene expression have been associated with susceptibility to $\mathrm{BU}^{76}$. Besides, mycolactone efficiently prevented production of cytokines and chemokines by activated monocytes and macrophages ${ }^{69,77-79}$ in vitro, with $\mathrm{IC}_{50}$ in the $5-20 \mathrm{nM}$ range. However, exposing macrophages to such mycolactone concentrations for $>48 \mathrm{~h}$ resulted in cytotoxicity ${ }^{69}$. In conclusion, whether produced by phagocytosed $M$. ulcerans or by clusters of extracellular bacteria, mycolactone potently impairs the antimicrobial and proinflammatory functions of infected macrophages, and eventually their viability.

In addition to being antigen-presenting cells, dendritic cells (DCs) are key to the initiation of primary immune responses. Addition of noncytotoxic concentrations of mycolactone to DCs limited their migratory properties and ability to mature and activate $\mathrm{T}$ cells in vitro ${ }^{80}$. In intradermally-injected mice, mycolactone also blocked the maturation and emigration of skin DCs to draining lymph nodes ${ }^{80}$. Endogenously-expressed and exogenously added mycolactone both inhibited DC's capacity to produce inflammatory chemokines upon activation with TLR ligands ${ }^{80}$. Together, these results suggested that bacterial production of mycolactone may limit both initiation of primary immune responses and recruitment of inflammatory cells to the infection site. In addition to presenting intracellular antigens in the context of MHC class II molecules, DCs can capture antigens released by surrounding cells and 
present them to $\mathrm{CD} 8^{+} \mathrm{T}$ cells in the context of $\mathrm{MHC}$ class I molecules, a process referred to as cross-presentation that leads to the activation of antigen-specific T cells. Mycolactone suppressed both direct and cross-presentation of synthetic peptides to $\mathrm{CD} 8^{+} \mathrm{T}$ cells, through inhibition of DC expression of several mediators of antigen presentation, including MHC class I and II ${ }^{81}$. Our proteomic profiling of mycolactone-DCs also revealed that mycolactone rapidly activates the Unfolded Protein Response (UPR) in the endoplasmic reticulum (ER), as reflected by transcriptional induction of Activating Transcription Factor 4 (ATF4) and its pro-apoptotic C/EBP Homologous Protein (CHOP) target gene ${ }^{68}$. Of note, mycolactone reproducibly reduced gene expression of the ER lumen chaperone BIP, a master regulator of the UPR, thus potentially increasing DC's susceptibility to ER stress-induced apoptosis ${ }^{68}$. In all, mycolactone interferes with DC immunobiology in multiple ways, which may account for the lack of inflammation in lesions and the defective cellular immune responses in patients with BU (see §IV).

Paradoxical reactions, resulting in clinical deterioration after initial improvement, are commonly observed in patients receiving an antibiotic treatment for $M$. ulcerans infection 17,20-22. Based on the lack of viable mycobacteria and evidence of significant inflammatory responses in excised tissues, $\mathrm{O}^{\prime}$ Brien et al. proposed that such paradoxical reactions may in fact represent adverse consequences of effective bacterial killing 17,20. Indeed, immunohistochemical analysis of skin lesions from BU patients receiving antibiotic treatment revealed massive leukocyte infiltration in the areas surrounding coagulative necrosis, including mononuclear phagocytes with intracellular mycobacteria and lymphocytes ${ }^{82,83}$. The paradoxical reactions developing in treated $\mathrm{BU}$ patients are reminiscent of the immune reconstitution inflammatory syndrome occurring in $M$. tuberculosis and HIV co-infected 
patients receiving anti-retroviral treatment, and the reactions occurring in leprosy patients receiving multi-drug therapy ${ }^{84}$. In $\mathrm{BU}$ patients, development of paradoxical reactions correlated with initially high bacterial load ${ }^{22}$. We can speculate that antibiotic treatment leads to the accumulation of pathogen-associated molecular patterns in BU lesions, which stimulate vigorous inflammatory responses upon reversal of local immune suppression by mycolactone. While consistent with the long-term persistence of mycolactone in treated patients, further studies of mycolactone and cytokine dynamics in lesional skin will be necessary to fully understand the immune mechanisms underpinning paradoxical reactions in BU patients receiving antibiotic therapy.

\section{IV - Mycolactone impact on adaptive immunity}

Little is known about the humoral response to $M$. ulcerans and its importance in protective immunity. While mice immunized with heat-killed bacteria rapidly developed an intense serum antibody response to $M$. ulcerans components, that of mice infected with live bacilli was limited and delayed ${ }^{85}$, suggesting that bacterial production of mycolactone suppresses the development of systemic humoral immune responses and/or antibody production by $B$ cells. B lymphocytes were reported to accumulate in the immune infiltrate surrounding the necrotic core of lesions from BU patients ${ }^{66}$, arguing in favor of the latter hypothesis. Immunoglobulins being either secreted or membrane proteins, mycolactone-mediated Sec61 blockade is predicted to inhibit their production by B cells (see $\S \mathrm{V}$ ). Yet, local production of $\operatorname{lgM}$, IgA and IgG responses, including mycolactone-neutralizing antibodies, was reported in 
M. ulcerans-infected mice ${ }^{86}$. Moreover, IgGs binding to mycolactone were identified in the skin biopsies of $60 \%$ of patients with PCR-confirmed BU, indicating that bacterial production of mycolactone is not be sufficient to stop recruited B cells to produce antigen-specific antibodies ${ }^{86}$. The number of antibody-producing cells increased in the skin of FVB/N mice during spontaneous healing, suggesting a role for a local production of anti-mycolactone antibodies in ulcer resolution ${ }^{86}$.

In BU patients with progressive ulcers, peripheral blood T cells showed defects in ability to produce cytokines including IFN- $\gamma$ upon ex vivo stimulation, irrespective of the activation stimuli ${ }^{87-91}$. Defective production of IFN- $\gamma$ by activated T cells resolved after surgical excision of the lesions, demonstrating their association with the presence of bacteria ${ }^{90}$. Early studies conducted by Foxwell and co-workers introduced the notion that mycolactone may be the bacterial factor causing these defects, by showing that exogenous addition of mycolactone prevents the production of IL-2 by activated T cell lines in vitro, in conditions not altering cell viability ${ }^{92}$. Subsequent studies using human peripheral blood $\mathrm{CD}^{+} \mathrm{T}$ lymphocytes showed that mycolactone's effects were not restricted to IL-2, as it also blocked efficiently the activation-induced production of IFN- $\gamma$, IL-4, IL-17, IL-10, TNF, IL-8 and MIP-1 ${ }^{91}$. Notably, mycolactone also reduced T cell expression of TCR and homing receptor L-selectin (CD62L), leading to impaired responsiveness to TCR stimulation ${ }^{62}$ and reduced capacity to reach peripheral lymph nodes in vivo ${ }^{69,93}$.

Generation of cellular immune responses is essential for protective immunity against most mycobacterial infections, and evidence suggests that $\mathrm{BU}$ is no exception. Observations that mycolactone potently suppresses innate and adaptive immune responses in vitro 
(summarized in Figure 2) suggests that ability to synthesize this factor was evolved by $M$. ulcerans to escape an efficacious host immune response. At the site of infection, active production of mycolactone by M. ulcerans maintain its local concentration at a high level, leading to apoptosis of tissue-resident cells and immune cell infiltrates. The belt of infiltrating leukocytes surrounding the necrotic core of BU lesions likely reflects a "war" zone where mycolactone concentrations paralyze and eventually kill immune cells, while failing to prevent their continuous influx. Part of the mycolactone produced by bacteria infecting the skin gains access to immune cells of peripheral blood and lymphoid organs to suppress the initiation of adaptive immune responses and effector functions of T lymphocytes ${ }^{6,24,94}$. The vigorous inflammatory responses occurring during antibiotic treatment suggest that BU patients are fully capable of reactivating cellular immune responses when bacterial production of mycolactone declines. Identifying patients at risk to develop paradoxical reactions, and better understanding the underlying immune mechanisms will help define personalized approaches to improve wound healing and clinical cure.

\section{V - Molecular target and mechanism of action}

Given its cytopathic effects in most cell types, mycolactone was initially considered a new type of bacterial toxin. However, lack of inflammation and pain in necrotic BU lesions on the one hand, and in vitro demonstration of its unique immunomodulatory properties of mycolactone on the other hand, suggested that mycolactone may also constitute, at non-cytotoxic doses, a novel type of natural immunosuppressor. Whether the immunomodulatory and cytotoxic 
properties of mycolactone were mechanistically linked, or resulted from interactions with distinct host receptors has remained elusive for decades.

In 2014, Simmonds and co-workers made a breakthrough with the demonstration that mycolactone prevents the translocation of secretory proteins into the ER, leading to their degradation in the cytosol by the ubiquitin:proteasome system ${ }^{78}$. Using cell-free systems, High et al. then showed that mycolactone selectively affects the cotranslational translocation of secretory proteins into the ER ${ }^{95}$. In eukaryotes cotranslational protein translocation is initiated by recognition of signal peptides or nascent polypeptide anchor domains by the signal recognition particle (SRP). The SRP then targets the ribosome-nascent polypeptide complex to the Sec61 translocon for insertion in the ER membrane (Figure 3). Sec61 is a heterotrimeric complex mediating the transport of secretory and integral transmembrane proteins (TMPs) into the ER. In collaboration with the group of Ville Paavilainen, we provided genetic evidence that mycolactone operates by directly targeting the pore-forming (alpha) subunit of the Sec61 translocon ${ }^{74}$. We identified single amino acid mutations in Sec61 not affecting the functionality of the Sec61 translocon, while fully protecting human cells against the cytotoxic and immunomodulatory activity of mycolactone ${ }^{74}$. Quantitative proteomics revealed that during T cell activation mycolactone-mediated inhibition of Sec61 $\alpha$ blunts the expression of both IFN- $\gamma$ cytokine and receptor, leading to defective induction of IFN- $\gamma$ inducible genes by autocrine signaling. Expression of mutant Sec61 $\alpha$ in mycolactone-treated T cells restored normal expression of IFN- $\gamma$ cytokine and receptor. Furthermore, when expressed in macrophages the mycolactone resistant mutant restored IFN- $\gamma$ receptormediated anti-microbial responses. These findings identified Sec61 as the host receptor mediating the immunomodulatory effects of mycolactone, and therefore the virulence of $M$. 
ulcerans. Moreover, they revealed the potential of inhibiting protein translocation for tuning down inflammatory and immune responses.

Other natural Sec61 blockers have been identified, which inhibit protein translocation by targeting a partially overlapping site in the pore-forming Sec61 $\alpha$ subunit ${ }^{96}$. Interestingly, the mutations conferring resistance to mycolactone clustered in the same region ${ }^{74}$, suggesting that structurally different Sec61 $\alpha$ inhibitors share a common binding site and mode of action. Gérard et al. recently reported an electron cryo-microscopy structure of mycolactone bound Sec61 $\alpha{ }^{97}$. Surprisingly, the mycolactone-binding pocket did not overlap with the region identified by resistance mutations, suggesting that such mutations may induce conformational changes indirectly preventing mycolactone binding. Mycolactone is composed of a lactone ring with two polyketide chains branched in the north and south positions (Figure 1). Mycolactone derivatives lacking the southern chain were unable to bind $\operatorname{Sec} 61 \alpha$ and were biologically inert ${ }^{69,74}$. In contrast, lactone core linked to southern chain retained both ability to bind to Sec61 $\alpha$ and mycolactone's immunosuppressive and cytotoxic properties ${ }^{69,74}$. This highlighted the importance of the lactone core/southern chain structural module in Sec61 $\alpha$ inhibition, but in the structure the southern polyketide chain of mycolactone had minimal interaction with $\operatorname{Sec} 61 \alpha^{96}$. Why the mycolactone binding site observed in the 3D-structure of Sec61 $\alpha$ is not that defined by the resistance mutations and mycolactone structure-activity relationship studies is unclear and it remains to be seen if allosteric conformational changes are involved.

Sec61 substrates include secretory and integral transmembrane proteins (TMPs), which can be divided into Type I, II or III according to the presence of a signal peptide (SP) and the 
orientation of the protein $\mathrm{N}$-terminus at the ER membrane. In cell-free systems, translocation of model secretory proteins, Type I and Type II TMPs was efficiently blocked by mycolactone 74,78,95. In contrast, mycolactone had no effect on the integration of Type III TMPs, a rare subset of TMPs where the transmembrane span acts as the ER targeting signal ${ }^{98}$. In accordance with in vitro findings, our profiling of mycolactone-susceptible proteome in CD4+ T lymphocytes, DCs and sensory neurons suggested that mycolactone is a multipotent, but not omnipotent Sec61 blocker $68,74,81$. Importantly, global proteomic analyses revealed that mycolactonemediated Sec61 blockade rapidly induces alterations beyond Sec61 substrates. In T cells for instance, mycolactone-mediated inhibition of IFN- $\gamma$ cytokine and receptor, both Sec61 substrates, was associated with defective transcriptional induction of IFN- $\gamma$-inducible genes, among which a range of cytosolic and nuclear proteins that are not Sec61 substrates. Concomitantly, Sec61 blockade triggered the upregulation of a subset of proteins involved in cellular stress responses ${ }^{68}$.

Over-expressing the R66G mutant of Sec61 $\alpha$ protected cells against mycolactone-mediated cell death, showing that its cytotoxicity strictly depends on mycolactone binding to Sec61 ${ }^{74}$. Our integrated analysis of mycolactone-driven proteomic alterations in T cells, DCs and neurons revealed the induction of cytosolic chaperones Hsp70/Hsp90 68. Moreover, mycolactone activated an atypical ER stress response, differing from the conventional unfolded protein response (UPR) by downregulation of the ER chaperone BIP (Figure 4). In contrast, Ogbechi et al. reported that mycolactone induces the integrated stress response (ISR) in treated macrophages and epithelial cell lines, in the absence of ER stress ${ }^{99}$. Whether primarily involving the ISR or the UPR, mycolactone-mediated stress responses were found in both studies to activate the pro-apoptotic ATF4/CHOP signaling pathway (Figure 4). In addition 
to revealing that Sec61 blockade by mycolactone induces proteostatic stress in the cytosol and the ER, these investigations provided an explanation for how sustained exposure to saturating amounts of mycolactone can lead to cell apoptosis.

\section{VI- Implications for BU prevention}

Attempts to generate a potent anti-BU vaccine have so far been unsuccessful. While reducing the duration of ulcers ${ }^{100}$ and risk of developing osteomyelitis, the most severe form of $\mathrm{BU}{ }^{101}$, the live attenuated anti-TB vaccine $M$. bovis Bacille Calmette-Guérin (BCG) only confers shortlasting protection against $B{ }^{102}$. Similar to $B C G$, a mycolactone-deficient strain of $M$. ulcerans delayed the onset of ulceration in mice experimentally infected in the footpads, but did not prevent disease progression ${ }^{103}$. The modest protective effect of BCG was not improved by booster vaccination in the mouse model ${ }^{104}$, arguing against multiple immunizations with BCG as a viable strategy for $\mathrm{BU}$ prevention. Perspectives for the development of better anti-BU vaccines include recombinant BCG strains expressing immunodominant $M$. ulcerans antigens and subunit-based vaccines ${ }^{105}$. DNA vaccines encoding $M$. ulcerans antigen $85 \mathrm{~A}, M$. leprae heat-shock protein Hsp65 or domains of the PKSs synthesizing mycolactone significantly reduced bacterial loads in $M$. ulcerans-infected mice ${ }^{106-109}$. However, they were less protective than BCG, even when administered in DNA prime-protein boost protocols.

Antibodies preventing mycolactone diffusion into host cells may help potentiate cellular immune responses to the pathogen. By immunizing mice with a detoxified version of 
mycolactone coupled to BSA as an immunogen, Plüschke and col. were able to generate antibodies preventing mycolactone-mediated cell apoptosis in vitro ${ }^{110}$. Human antibodies recognizing mycolactone were successfully isolated from naïve phage and yeast display random libraries, and affinity matured by error prone PCR ${ }^{111}$. These results illustrate the potential of synthetic mycolactone derivatives to act as anti-BU vaccines, and also the possibility of generating anti-mycolactone antibodies for research, diagnosis and potentially treatment of BU. However, additional work will be required to determine whether antimycolactone antibodies neutralize or exacerbate its toxicity in vivo.

\section{VII- Therapeutic potential of mycolactone}

Whether released by bacteria infecting the skin or injected via the subcutaneous route, mycolactone was found to diffuse into the leukocytes of peripheral blood and lymphoid organs 52,56 . With this distribution profile, the unique immunomodulatory effects of mycolactone make it a potential drug candidate for therapeutic use against inflammatory disorders. Using mouse models, we showed that systematically delivered mycolactone confers protection against chronic skin inflammation, rheumatoid arthritis and inflammatory pain ${ }^{69}$. However, the low yields and complexity of chemical schemes for mycolactone synthesis make its large-scale production for clinical applications challenging 45,112,113. Structure-activity relationship studies showed that the mycolactone subunit corresponding to lactone core and southern polyketide chain is critical for binding to Sec61 and biological activity $69,74,114$. While less potent than natural mycolactone, the truncated version retained capacity to block cytokine production by neutrophils, macrophages and lymphocytes in vitro 
and efficiently protected mice against inflammatory disorders and pain in mouse models ${ }^{69}$. From a clinical perspective, this chemically simpler version of mycolactone offers several advantages over the natural molecule as in addition to being easier to synthesize it displayed a better therapeutic window in vivo.

Many pathogenic human viruses rely on host biosynthetic machineries to produce the viral proteins required for replication, suggesting that Sec61 blockade could be used as a strategy to block viral propagation. In support of this hypothesis, mycolactone-mediated inhibition of Sec61 efficiently prevented host cell production of influenza A virus HA and NA proteins 68. Inhibiting Sec61 with mycolactone in ZIKA virus-infected cells blocked vacuole formation and virus production ${ }^{115}$. It significantly delayed virus propagation and lethality in IFNAR ${ }^{-/-}$mice (personal communication). Beyond its direct antiviral effects, mycolactone-mediated Sec61 blockade may prevent the pathology that is associated with virus-driven inflammation. Since mycolactone dose-dependently inhibited the infectivity of Sars-Cov-2 in conditions not affecting host cell viability in vitro (personal communication), it will be interesting to determine if it blocks viral propagation and pathological inflammation in vivo.

There is epidemiological evidence suggesting a higher incidence of HIV infection in BU patients and a stimulatory effect of HIV infection on BU severity ${ }^{116-118}$. Although HIV-M. ulcerans coinfection is a rare event, these studies highlight the need to elucidate the immunological events underpinning disease association ${ }^{119}$. While mycolactone-mediated inhibition of Sec61 strongly reduced HIV-1 envelope synthesis and viral infectivity in vitro (personal communication), its suppressive effects on the development of Type I IFN-mediated antiviral responses may override its direct antiviral activity. 
Finally, since cancer cells rely on active protein translocation into the ER for fast growth, Sec61 inhibitors like mycolactone may show a potential as anticancer drugs ${ }^{120}$. Mycolactone at nanomolar concentrations induced apoptosis in several cancer cell lines, likely through induction of proteotoxic stress responses ${ }^{121}$. It depleted the UPR regulator BIP in treated cell lines 68,74 , thereby potentially accelerating stress-induced apoptosis. Whether these effects operate in primary tumors and in mouse xenograft models remains to be established.

\section{Conclusion}

Through the study of mycolactone, research on M. ulcerans infection has identified a novel mechanism of immune suppression based on protein translocation blockade. Mycolactonemediated inhibition of Sec61 not only reveals the so far unanticipated potential of inhibiting Sec61 for immune modulation, it provides a mechanism for $M$. ulcerans virulence and BU pathogenesis. Nonetheless, a large number of unresolved issues remain, particularly in relation to immunity against $M$. ulcerans. Future challenges will consist in defining a vaccine conferring solid and long-term protection against BU, predicting and preventing paradoxical reactions in treated patients, and identifying means to reduce the use of antibiotics by neutralizing the immunosuppressive action of mycolactone. 


\section{Acknowledgements}

C.D. acknowledges core support from Institut Pasteur and INSERM.

\section{References}

1. Yotsu RR, Suzuki K, Simmonds RE, et al. Buruli Ulcer: a Review of the Current Knowledge. Curr Trop Med Reports. 2018. doi:10.1007/s40475-018-0166-2

2. Pluschke G, Röltgen K. Epidemiology and disease burden of Buruli ulcer: a review. Res Rep Trop Med. 2015. doi:10.2147/rrtm.s62026

3. Huang GKL, Johnson PD. Epidemiology and management of Buruli ulcer. Expert Rev Anti Infect Ther. 2014. doi:10.1586/14787210.2014.910113

4. Simpson H, Deribe K, Tabah EN, et al. Mapping the global distribution of Buruli ulcer: a systematic review with evidence consensus. Lancet Glob Heal. 2019. doi:10.1016/S2214-109X(19)30171-8

5. Omansen TF, Erbowor-Becksen A, Yotsu R, et al. Global epidemiology of Buruli ulcer, 2010-2017, and analysis of 2014 WHO programmatic targets. Emerg Infect Dis. 2019. doi:10.3201/eid2512.190427

6. Guenin-Macé L, Ruf M-T, Pluschke G, Demangel C. Mycolactone: More than Just a Cytotoxin. In: Buruli Ulcer. ; 2019. doi:10.1007/978-3-030-11114-4_7 
7. Guarner J. Buruli ulcer. Review of a neglected skin mycobacterial disease. J Clin Microbiol. 2018. doi:10.1128/JCM.01507-17

8. Abass KM, van der Werf TS, Phillips RO, et al. Buruli ulcer control in a highly endemic district in Ghana: role of community-based surveillance volunteers. Am J Trop Med Hyg. 2015;92(1):115-117. doi:10.4269/ajtmh.14-0405

9. Röltgen K, Cruz I, Ndung'u JM, Pluschke G. Laboratory diagnosis of buruli ulcer: Challenges and future perspectives. In: Buruli Ulcer: Mycobacterium Ulcerans Disease. ; 2019. doi:10.1007/978-3-030-11114-4_10

10. Stinear T, Ross BC, Davies JK, et al. Identification and characterization of IS2404 and IS2606: two distinct repeated sequences for detection of Mycobacterium ulcerans by PCR. J Clin Microbiol. 1999;37(4):1018-1023.

http://www.ncbi.nlm.nih.gov/entrez/query.fcgi?cmd=Retrieve\&db=PubMed\&dopt=Ci tation\&list_uids=10074520.

11. Phillips R, Horsfield C, Kuijper S, et al. Sensitivity of PCR targeting the IS2404 insertion sequence of Mycobacterium ulcerans in an Assay using punch biopsy specimens for diagnosis of Buruli ulcer. J Clin Microbiol. 2005;43(8):3650-3656.

http://www.ncbi.nlm.nih.gov/entrez/query.fcgi?cmd=Retrieve\&db=PubMed\&dopt=Ci tation\&list_uids=16081892.

12. Beissner M, Phillips RO, Battke F, et al. Loop-Mediated Isothermal Amplification for Laboratory Confirmation of Buruli Ulcer Disease-Towards a Point-of-Care Test. PLoS Negl Trop Dis. 2015;9(11):e0004219. doi:10.1371/journal.pntd.0004219

13. Omansen TF, van der Werf TS, Phillips RO. Antimicrobial treatment of mycobacterium ulcerans infection. In: Buruli Ulcer: Mycobacterium Ulcerans Disease. ; 2019. doi:10.1007/978-3-030-11114-4_11 
14. Beissner M, Awua-Boateng NY, Thompson W, et al. A genotypic approach for detection, identification, and characterization of drug resistance in Mycobacterium ulcerans in clinical samples and isolates from Ghana. Am J Trop Med Hyg. 2010. doi:10.4269/ajtmh.2010.10-0263

15. Phillips RO, Robert J, Abass KM, et al. Rifampicin and clarithromycin (extended release) versus rifampicin and streptomycin for limited Buruli ulcer lesions: a randomised, open-label, non-inferiority phase 3 trial. Lancet. 2020. doi:10.1016/S0140-6736(20)30047-7

16. Paul D R Johnson. Buruli ulcer: cured by 8 weeks of oral antibiotics? Lancet. https://www.thelancet.com/journals/lancet/article/PIIS0140-6736(20)304785/fulltext.

17. O'Brien DP, Robson ME, Callan PP, McDonald AH. "Paradoxical" immune-mediated reactions to Mycobacterium ulcerans during antibiotic treatment: A result of treatment success, not failure. Med J Aust. 2009. doi:10.5694/j.13265377.2009.tb03313.x

18. Nienhuis WA, Stienstra Y, Abass KM, et al. Paradoxical responses after start of antimicrobial treatment in mycobacterium ulcerans infection. Clin Infect Dis. 2012. doi:10.1093/cid/cir856

19. Ruf MT, Chauty A, Adeye A, et al. Secondary buruli ulcer skin lesions emerging several months after completion of chemotherapy: Paradoxical reaction or evidence for immune protection? PLoS Neg/ Trop Dis. 2011. doi:10.1371/journal.pntd.0001252

20. O'Brien DP, Robson M, Friedman ND, et al. Incidence, clinical spectrum, diagnostic features, treatment and predictors of paradoxical reactions during antibiotic treatment of Mycobacterium ulcerans infections. BMC Infect Dis. 2013. 
doi:10.1186/1471-2334-13-416

21. Barogui YT, Klis SA, Johnson RC, et al. Genetic Susceptibility and Predictors of Paradoxical Reactions in Buruli Ulcer. PLoS Negl Trop Dis. 2016.

doi:10.1371/journal.pntd.0004594

22. Frimpong $\mathrm{M}$, Agbavor $\mathrm{B}$, Duah MS, et al. Paradoxical reactions in Buruli ulcer after initiation of antibiotic therapy: Relationship to bacterial load. PLoS Neg/ Trop Dis. 2019. doi:10.1371/journal.pntd.0007689

23. Vandelannoote K, Eddyani M, Buultjens A, Stinear TP. Population genomics and molecular epidemiology of mycobacterium ulcerans. In: Buruli Ulcer: Mycobacterium Ulcerans Disease. ; 2019. doi:10.1007/978-3-030-11114-4_6

24. Demangel C, Stinear TP, Cole ST. Buruli ulcer: reductive evolution enhances pathogenicity of Mycobacterium ulcerans. Nat Rev Microbiol. 2009;7(1):50-60. doi:nrmicro2016 [pii]10.1038/nrmicro2077

25. Yip MJ, Porter JL, Fyfe JA, et al. Evolution of Mycobacterium ulcerans and other mycolactone-producing mycobacteria from a common Mycobacterium marinum progenitor. J Bacteriol. 2007;189(5):2021-2029. doi:JB.01442-06 [pii]10.1128/JB.01442-06

26. Doig KD, Holt KE, Fyfe JA, et al. On the origin of Mycobacterium ulcerans, the causative agent of Buruli ulcer. BMC Genomics. 2012;13:258. doi:10.1186/1471-2164$13-258$

27. Stinear TP, Seemann T, Pidot S, et al. Reductive evolution and niche adaptation inferred from the genome of Mycobacterium ulcerans, the causative agent of Buruli ulcer. Genome Res. 2007;17(2):192-200. doi:gr.5942807 [pii]10.1101/gr.5942807

28. Hong H, Demangel C, Pidot SJ, Leadlay PF, Stinear T. Mycolactones: 
immunosuppressive and cytotoxic polyketides produced by aquatic mycobacteria. Nat Prod Rep. 2008;25(3):447-454. doi:10.1039/b803101k

29. Merritt RW, Walker ED, Small PLC, et al. Ecology and transmission of buruli ulcer disease: A systematic review. PLoS Negl Trop Dis. 2010.

doi:10.1371/journal.pntd.0000911

30. Eddyani M, Portaels F. Survival of Mycobacterium ulcerans at 37 degrees C. Clin Microbiol Infect. 2007;13(10):1033-1035.

31. Sexton-Oates NK, Stewardsonid AJ, Yerramilli A, Johnson PDR. Does skin surface temperature variation account for buruli ulcer lesion distribution? PLoS Negl Trop Dis. 2020. doi:10.1371/journal.pntd.0007732

32. O'Brien DP, Wynne JW, Buultjens AH, et al. Exposure Risk for Infection and Lack of Human-to-Human Transmission of Mycobacterium ulcerans Disease, Australia. Emerg Infect Dis. 2017;23(5):837-840. doi:10.3201/eid2305.160809

33. Jacobsen $\mathrm{KH}$, Padgett JJ. Risk factors for Mycobacterium ulcerans infection. Int J Infect Dis. 2010. doi:10.1016/j.ijid.2009.11.013

34. Vandelannooteid K, Pluschke G, Bolz M, et al. Introduction of Mycobacterium ulcerans disease in the Bankim health district of Cameroon follows damming of the mape river. PLoS Negl Trop Dis. 2020. doi:10.1371/journal.pntd.0008501

35. Combe M, Velvin CJ, Morris A, et al. Global and local environmental changes as drivers of Buruli ulcer emergence. Emerg Microbes Infect. 2017;6(5):e34. doi:10.1038/emi.2017.45

36. Zingue D, Bouam A, Tian RBD, Drancourt M. Buruli Ulcer, a Prototype for EcosystemRelated Infection, Caused by Mycobacterium ulcerans. Clin Microbiol Rev. 2018;31(1). doi:10.1128/CMR.00045-17 
37. Marsollier L, Aubry J, Coutanceau E, et al. Colonization of the salivary glands of Naucoris cimicoides by Mycobacterium ulcerans requires host plasmatocytes and a macrolide toxin, mycolactone. Cell Microbiol. 2005;7(7):935-943.

http://www.ncbi.nlm.nih.gov/entrez/query.fcgi?cmd=Retrieve\&db=PubMed\&dopt=Ci tation\&list_uids=15953026.

38. Wallace JR, Mangas KM, Porter JL, et al. Mycobacterium ulcerans low infectious dose and mechanical transmission support insect bites and puncturing injuries in the spread of Buruli ulcer. PLoS Negl Trop Dis. 2017;11(4):e0005553. doi:10.1371/journal.pntd.0005553

39. Williamson HR, Benbow ME, Nguyen KD, et al. Distribution of Mycobacterium ulcerans in Buruli ulcer endemic and non-endemic aquatic sites in Ghana. PLoS Negl Trop Dis. 2008. doi:10.1371/journal.pntd.0000205

40. Fyfe JAM, Lavender $\mathrm{CJ}$, Handasyde KA, et al. A major role for mammals in the ecology of Mycobacterium ulcerans. PLoS Negl Trop Dis. 2010. doi:10.1371/journal.pntd.0000791

41. Marsollier L, Sévérin T, Aubry J, et al. Aquatic snails, passive hosts of Mycobacterium ulcerans. Appl Environ Microbiol. 2004. doi:10.1128/AEM.70.10.6296-6298.2004

42. George KM, Chatterjee D, Gunawardana G, et al. Mycolactone: a polyketide toxin from Mycobacterium ulcerans required for virulence. Science (80- ). 1999;283(5403):854-857. http://www.ncbi.nIm.nih.gov/entrez/query.fcgi?cmd=Retrieve\&db=PubMed\&dopt=Ci tation\&list_uids=9933171.

43. Jenkin GA, Stinear TP, Johnson PD, Davies JK. Subtractive hybridization reveals a type I polyketide synthase locus specific to Mycobacterium ulcerans. J Bacteriol. 
2003;185(23):6870-6882.

http://www.ncbi.nlm.nih.gov/entrez/query.fcgi?cmd=Retrieve\&db=PubMed\&dopt=Ci tation\&list_uids=14617651.

44. Stinear TP, Mve-Obiang A, Small PL, et al. Giant plasmid-encoded polyketide synthases produce the macrolide toxin of Mycobacterium ulcerans. Proc Natl Acad Sci U SA. 2004;101(5):1345-1349. doi:10.1073/pnas.03058771010305877101 [pii]

45. Gehringer M, Altmann KH. The chemistry and biology of mycolactones. Beilstein J Org Chem. 2017;13:1596-1660. doi:10.3762/bjoc.13.159

46. Pidot SJ, Asiedu K, Kaser M, Fyfe JA, Stinear TP. Mycobacterium ulcerans and other mycolactone-producing mycobacteria should be considered a single species. PLoS Negl Trop Dis. 2010;4(7):e663. doi:10.1371/journal.pntd.0000663

47. Kishi Y. Chemistry of mycolactones, the causative toxins of Buruli ulcer. Proc Natl Acad Sci U S A. 2011;108(17):6703-6708. doi:10.1073/pnas.1015252108

48. Mve-Obiang A, Lee RE, Portaels F, Small PLC. Heterogeneity of mycolactones produced by clinical isolates of Mycobacterium ulcerans: Implications for virulence. Infect Immun. 2003;71(2):774-783.

49. Marsollier L, Brodin P, Jackson M, et al. Impact of Mycobacterium ulcerans biofilm on transmissibility to ecological niches and Buruli ulcer pathogenesis. PLoS Pathog. 2007;3(5):e62. doi:06-PLPA-RA-0152R2 [pii]10.1371/journal.ppat.0030062

50. Tobias NJ, Seemann T, Pidot SJ, et al. Mycolactone gene expression is controlled by strong SigA-like promoters with utility in studies of Mycobacterium ulcerans and buruli ulcer. PLoS Neg/ Trop Dis. 2009;3(11):e553. doi:10.1371/journal.pntd.0000553

51. Sarfo FS, Phillips RO, Zhang J, et al. Kinetics of mycolactone in human subcutaneous tissue during antibiotic therapy for Mycobacterium ulcerans disease. BMC Infect Dis. 
2014;14(1):202. doi:10.1186/1471-2334-14-202

52. Sarfo FS, Chevalier F, Aka N, et al. Mycolactone diffuses into the peripheral blood of buruli ulcer patients - implications for diagnosis and disease monitoring. PLoS Negl Trop Dis. 2011;5(7):e1237. doi:10.1371/journal.pntd.0001237

53. Deshayes C, Angala SK, Marion E, et al. Regulation of Mycolactone, the Mycobacterium ulcerans Toxin, Depends on Nutrient Source. PLoS Negl Trop Dis. 2013. doi:10.1371/journal.pntd.0002502

54. Porter JL, Tobias NJ, Pidot SJ, et al. The Cell Wall-Associated Mycolactone Polyketide Synthases Are Necessary but Not Sufficient for Mycolactone Biosynthesis. PLoS One. 2013;8(7). doi:10.1371/journal.pone.0070520

55. Kubicek-Sutherland JZ, Vu DM, Anderson AS, et al. Understanding the significance of biochemistry in the storage, handling, purification, and sampling of amphiphilic mycolactone. Toxins (Basel). 2019. doi:10.3390/toxins11040202

56. Hong H, Coutanceau E, Leclerc M, Caleechurn L, Leadlay PF, Demangel C. Mycolactone Diffuses from Mycobacterium ulcerans-Infected Tissues and Targets Mononuclear Cells in Peripheral Blood and Lymphoid Organs. PLoS Negl Trop Dis. 2008;2(10):e325. http://www.ncbi.nlm.nih.gov/entrez/query.fcgi?cmd=Retrieve\&db=PubMed\&dopt=Ci tation\&list_uids=18941518.

57. Colucci-Guyon E, Rifflet A, Saint-Auret S, et al. Spatiotemporal analysis of mycolactone distribution in vivo reveals partial diffusion in the central nervous system. PLoS Negl Trop Dis. 2020;14(12).

58. Guarner J. Buruli ulcer. Review of a neglected skin mycobacterial disease. J Clin Microbiol. 2018. doi:10.1128/JCM.01507-17

59. Snyder DS, Small PL. Uptake and cellular actions of mycolactone, a virulence 
determinant for Mycobacterium ulcerans. Microb Pathog. 2003;34(2):91-101.

http://www.ncbi.nlm.nih.gov/entrez/query.fcgi?cmd=Retrieve \&db=PubMed\&dopt=Ci tation\&list_uids=12623277.

60. López CA, Unkefer CJ, Swanson BI, Swanson JMJ, Gnanakaran S. Membrane perturbing properties of toxin mycolactone from Mycobacterium ulcerans. PLoS Comput Biol. 2018;14(2):e1005972. doi:10.1371/journal.pcbi.1005972

61. Nitenberg M, Benarouche A, Maniti O, et al. The potent effect of mycolactone on lipid membranes. PLoS Pathog. 2018;14(1):e1006814. doi:10.1371/journal.ppat.1006814

62. Boulkroun S, Guenin-Mace L, Thoulouze MI, et al. Mycolactone suppresses T cell responsiveness by altering both early signaling and posttranslational events. $J$ Immunol. 2010;184(3):1436-1444. doi:jimmunol.0902854 [pii]10.4049/jimmunol.0902854

63. Coutanceau E, Marsollier L, Brosch R, et al. Modulation of the host immune response by a transient intracellular stage of Mycobacterium ulcerans: the contribution of endogenous mycolactone toxin. Cell Microbiol. 2005;7(8):1187-1196. http://www.ncbi.nlm.nih.gov/entrez/query.fcgi?cmd=Retrieve\&db=PubMed\&dopt=Ci tation\&list_uids=16008585.

64. Oliveira MS, Fraga AG, Torrado E, et al. Infection with Mycobacterium ulcerans induces persistent inflammatory responses in mice. Infect Immun. 2005;73(10):62996310. doi:73/10/6299 [pii]10.1128/IAI.73.10.6299-6310.2005

65. Torrado E, Fraga AG, Castro AG, et al. Evidence for an intramacrophage growth phase of Mycobacterium ulcerans. Infect Immun. 2007;75(2):977-987. doi:IAl.00889-06 [pii]10.1128/IAI.00889-06

66. Ruf MT, Steffen C, Bolz M, Schmid P, Pluschke G. Infiltrating leukocytes surround early 
Buruli ulcer lesions, but are unable to reach the mycolactone producing mycobacteria. Virulence. 2017;8(8):1918-1926. doi:10.1080/21505594.2017.1370530

67. De Oliveira S, Rosowski EE, Huttenlocher A. Neutrophil migration in infection and wound repair: Going forward in reverse. Nat Rev Immunol. 2016.

doi:10.1038/nri.2016.49

68. Morel JD, Paatero AO, Wei J, et al. Proteomics reveals scope of mycolactone-mediated sec61 blockade and distinctive stress signature. Mol Cell Proteomics. 2018;17(9):17501765. doi:10.1074/mcp.RA118.000824

69. Guenin-Mace L, Baron L, Chany AC, et al. Shaping mycolactone for therapeutic use against inflammatory disorders. Sci Transl Med. 2015;7(289):289ra85. doi:10.1126/scitranslmed.aab0458

70. Tecchio C, Micheletti A, Cassatella MA. Neutrophil-derived cytokines: Facts beyond expression. Front Immunol. 2014. doi:10.3389/fimmu.2014.00508

71. Röltgen K, Pluschke G. Buruli ulcer: The Efficacy of Innate Immune Defense May Be a Key Determinant for the Outcome of Infection With Mycobacterium ulcerans. Front Microbiol. 2020. doi:10.3389/fmicb.2020.01018

72. Flynn JL, Chan J. Immunology of tuberculosis. Annu Rev Immunol. 2001;19:93-129. doi:10.1146/annurev.immunol.19.1.93

73. Bieri R, Bolz M, Ruf MT, Pluschke G. Interferon-gamma Is a Crucial Activator of Early Host Immune Defense against Mycobacterium ulcerans Infection in Mice. PLoS NegI Trop Dis. 2016;10(2):e0004450. doi:10.1371/journal.pntd.0004450

74. Baron L, Paatero AO, Morel JD, et al. Mycolactone subverts immunity by selectively blocking the Sec61 translocon. J Exp Med. 2016;213(13):2885-2896. doi:10.1084/jem.20160662 
75. Torrado E, Fraga AG, Logarinho E, et al. IFN- $\gamma$-Dependent Activation of Macrophages during Experimental Infections by Mycobacterium ulcerans Is Impaired by the Toxin Mycolactone .J Immunol. 2010. doi:10.4049/jimmunol.0902717

76. Bibert S, Bratschi MW, Aboagye SY, et al. Susceptibility to Mycobacterium ulcerans Disease (Buruli ulcer) Is Associated with IFNG and iNOS Gene Polymorphisms. Front Microbiol. 2017;8:1903. doi:10.3389/fmicb.2017.01903

77. Simmonds RE, Lali F V, Smallie T, Small PL, Foxwell BM. Mycolactone inhibits monocyte cytokine production by a posttranscriptional mechanism. J Immunol. 2009;182(4):2194-2202.

http://www.ncbi.nlm.nih.gov/entrez/query.fcgi?cmd=Retrieve \&db=PubMed\&dopt=Ci tation\&list_uids=19201873.

78. Hall BS, Hill K, McKenna M, et al. The pathogenic mechanism of the Mycobacterium ulcerans virulence factor, mycolactone, depends on blockade of protein translocation into the ER. PLoS Pathog. 2014;10(4):e1004061. doi:10.1371/journal.ppat.1004061

79. Sarfo FS, Phillips R, Wansbrough-Jones M, Simmonds RE. Recent advances: Role of mycolactone in the pathogenesis and monitoring of Mycobacterium ulcerans infection/Buruli ulcer disease. Cell Microbiol. 2016;18(1):17-29. doi:10.1111/cmi.12547

80. Coutanceau E, Decalf J, Martino A, et al. Selective suppression of dendritic cell functions by Mycobacterium ulcerans toxin mycolactone. J Exp Med. 2007;204(6):1395-1403. http://www.ncbi.nlm.nih.gov/entrez/query.fcgi?cmd=Retrieve\&db=PubMed\&dopt=Ci tation\&list_uids=17517970.

81. Grotzke JE, Kozik P, Morel JD, et al. Sec61 blockade by mycolactone inhibits antigen 
cross-presentation independently of endosome-to-cytosol export. Proc Natl Acad Sci U S A. 2017. doi:10.1073/pnas.1705242114

82. Schutte D, Um-Boock A, Mensah-Quainoo E, Itin P, Schmid P, Pluschke G.

Development of highly organized lymphoid structures in Buruli ulcer lesions after treatment with rifampicin and streptomycin. PLoS Negl Trop Dis. 2007;1(1):e2. doi:10.1371/journal.pntd.0000002

83. Schütte D, UmBoock A, Pluschke G. Phagocytosis of Mycobacterium ulcerans in the course of rifampicin and streptomycin chemotherapy in Buruli ulcer lesions. $\mathrm{Br} J$ Dermatol. 2009. doi:10.1111/j.1365-2133.2008.08879.x

84. Geluk A. Correlates of immune exacerbations in leprosy. Semin Immunol. 2018. doi:10.1016/j.smim.2018.06.003

85. Marion E, Jarry U, Cano C, et al. FVB/N Mice Spontaneously Heal Ulcerative Lesions Induced by Mycobacterium ulcerans and Switch M. ulcerans into a Low Mycolactone Producer . J Immunol. 2016. doi:10.4049/jimmunol.1502194

86. Foulon M, Pouchin A, Manry J, et al. Skin-specific antibodies neutralizing mycolactone toxin during the spontaneous healing of Mycobacterium ulcerans infection. Sci Adv. 2020. doi:10.1126/sciadv.aax7781

87. Gooding TM, Johnson PD, Campbell DE, et al. Immune response to infection with Mycobacterium ulcerans. Infect Immun. 2001;69(3):1704-1707. http://www.ncbi.nlm.nih.gov/entrez/query.fcgi?cmd=Retrieve\&db=PubMed\&dopt=Ci tation\&list_uids=11179346.

88. Prevot G, Bourreau E, Pascalis H, et al. Differential production of systemic and intralesional gamma interferon and interleukin-10 in nodular and ulcerative forms of Buruli disease. Infect Immun. 2004;72(2):958-965. 
http://www.ncbi.nlm.nih.gov/entrez/query.fcgi?cmd=Retrieve\&db=PubMed\&dopt=Ci tation\&list_uids=14742541.

89. Westenbrink BD, Stienstra Y, Huitema MG, et al. Cytokine responses to stimulation of whole blood from patients with buruli ulcer disease in Ghana. Clin Diagn Lab Immunol. 2005;12(1):125-129.

90. Yeboah-Manu D, Peduzzi E, Mensah-Quainoo E, et al. Systemic suppression of interferon-gamma responses in Buruli ulcer patients resolves after surgical excision of the lesions caused by the extracellular pathogen Mycobacterium ulcerans. J Leukoc Biol. 2006;79(6):1150-1156. doi:jlb.1005581 [pii]10.1189/jlb.1005581

91. Phillips R, Sarfo FS, Guenin-Mace L, et al. Immunosuppressive Signature of Cutaneous Mycobacterium ulcerans Infection in the Peripheral Blood of Patients with Buruli Ulcer Disease. J Infect Dis. 2009.

http://www.ncbi.nlm.nih.gov/entrez/query.fcgi?cmd=Retrieve\&db=PubMed\&dopt=Ci tation\&list_uids=19863437.

92. Pahlevan AA, Wright DJ, Andrews C, George KM, Small PL, Foxwell BM. The inhibitory action of Mycobacterium ulcerans soluble factor on monocyte/T cell cytokine production and NF-kappa B function. J Immunol. 1999;163(7):3928-3935. doi:ji_v163n7p3928 [pii]

93. Guenin-Mace L, Carrette F, Asperti-Boursin F, et al. Mycolactone impairs T cell homing by suppressing microRNA control of L-selectin expression. Proc Natl Acad Sci U S A. 2011;108(31):12833-12838. doi:1016496108 [pii]10.1073/pnas.1016496108

94. Coutanceau E, Decalf J, Martino A, et al. Selective suppression of dendritic cell functions by Mycobacterium ulcerans toxin mycolactone. J Exp Med. 2007;204(6):1395-1403. 
http://www.ncbi.nlm.nih.gov/entrez/query.fcgi?cmd=Retrieve\&db=PubMed\&dopt=Ci tation\&list_uids=17517970.

95. McKenna M, Simmonds RE, High S. Mechanistic insights into the inhibition of Sec61dependent co- and post-translational translocation by mycolactone. J Cell Sci. 2016. doi:10.1242/jcs.182352

96. Luesch H, Paavilainen VO. Natural products as modulators of eukaryotic protein secretion. Nat Prod Rep. 2020. doi:10.1039/c9np00066f

97. Gérard SF, Hall BS, Zaki AM, et al. Structure of the Inhibited State of the Sec Translocon. Mol Cell. 2020. doi:10.1016/j.molcel.2020.06.013

98. McKenna M, Simmonds RE, High S. Mycolactone reveals the substrate-driven complexity of Sec61-dependent transmembrane protein biogenesis. J Cell Sci. 2017;130(7):1307-1320. doi:10.1242/jcs.198655

99. Ogbechi J, Hall BS, Sbarrato T, et al. Inhibition of Sec61-dependent translocation by mycolactone uncouples the integrated stress response from ER stress, driving cytotoxicity via translational activation of ATF4. Cell Death Dis. 2018;9(3):397. doi:10.1038/s41419-018-0427-y

100. Amofah GK, Adjei-Acquah C, Sagoe-Moses C, Frimpong EH. Epidemiology of buruli ulcer in amansie west district, ghana. Trans R Soc Trop Med Hyg. 1993. doi:10.1016/0035-9203(93)90272-R

101. Portaels F, Aguiar J, Debacker M, et al. Mycobacterium bovis BCG Vaccination as Prophylaxis against Mycobacterium ulcerans Osteomyelitis in Buruli Ulcer Disease. Infect Immun. 2004. doi:10.1128/IAI.72.1.62-65.2004

102. Smith PG, Revill WD, Lukwago E, Rykushin YP. The protective effect of BCG against Mycobacterium ulcerans disease: a controlled trial in an endemic area of Uganda. 
Trans R Soc Trop Med Hyg. 1977;70(5-6):449-457.

http://www.ncbi.nlm.nih.gov/entrez/query.fcgi?cmd=Retrieve \&db=PubMed\&dopt=Ci tation\&list_uids=841647.

103. Fraga AG, Martins TG, Torrado E, et al. Cellular immunity confers transient protection in experimental Buruli ulcer following BCG or mycolactone-negative Mycobacterium ulcerans vaccination. PLoS One. 2012;7(3):e33406. doi:10.1371/journal.pone.0033406

104. Tanghe A, Adnet PY, Gartner T, Huygen K. A booster vaccination with Mycobacterium bovis BCG does not increase the protective effect of the vaccine against experimental Mycobacterium ulcerans infection in mice. Infect Immun. 2007;75(5):2642-2644. http://www.ncbi.nlm.nih.gov/entrez/query.fcgi?cmd=Retrieve\&db=PubMed\&dopt=Ci tation\&list_uids=17325046.

105. Einarsdottir T, Huygen K. Buruli ulcer. Hum Vaccin. 2011;7(11):1198-1203. doi:10.4161/hv.7.11.17751

106. Coutanceau E, Legras P, Marsollier L, Reysset G, Cole ST, Demangel C. Immunogenicity of Mycobacterium ulcerans Hsp65 and protective efficacy of a Mycobacterium leprae Hsp65-based DNA vaccine against Buruli ulcer. Microbes Infect. 2006;8(8):2075-2081. doi:10.1016/j.micinf.2006.03.009

107. Tanghe A, Content J, Van Vooren JP, Portaels F, Huygen K. Protective efficacy of a DNA vaccine encoding antigen 85A from Mycobacterium bovis BCG against Buruli ulcer. Infect Immun. 2001;69(9):5403-5411. http://www.ncbi.nlm.nih.gov/entrez/query.fcgi?cmd=Retrieve\&db=PubMed\&dopt=Ci tation\&list_uids=11500410.

108. Tanghe A, Dangy JP, Pluschke G, Huygen K. Improved protective efficacy of a speciesspecific DNA vaccine encoding mycolyl-transferase Ag85A from Mycobacterium 
ulcerans by homologous protein boosting. PLoS Negl Trop Dis. 2008.

doi:10.1371/journal.pntd.0000199

109. Roupie V, Pidot SJ, Einarsdottir T, et al. Analysis of the Vaccine Potential of Plasmid DNA Encoding Nine Mycolactone Polyketide Synthase Domains in Mycobacterium ulcerans Infected Mice. PLoS Neg/ Trop Dis. 2014. doi:10.1371/journal.pntd.0002604

110. Dangy JP, Scherr N, Gersbach P, et al. Antibody-Mediated Neutralization of the Exotoxin Mycolactone, the Main Virulence Factor Produced by Mycobacterium ulcerans. PLoS Negl Trop Dis. 2016;10(6):e0004808. doi:10.1371/journal.pntd.0004808

111. Naranjo L, Ferrara F, Blanchard N, et al. Recombinant Antibodies against Mycolactone. Toxins (Basel). 2019. doi:10.3390/toxins11060346

112. Saint-Auret S, Chany AC, Casarotto V, et al. Total Syntheses of Mycolactone A/B and its Analogues for the Exploration of the Biology of Buruli Ulcer. Chim. 2017;71(12):836-840. doi:10.2533/chimia.2017.836

113. Song F, Fidanze S, Benowitz AB, Kishi Y. Total synthesis of mycolactones A and B. Tetrahedron. 2007. doi:10.1016/j.tet.2007.02.057

114. Scherr N, Gersbach P, Dangy JP, et al. Structure-Activity Relationship Studies on the Macrolide Exotoxin Mycolactone of Mycobacterium ulcerans. PLoS Negl Trop Dis. 2013;7(3):e2143. doi:10.1371/journal.pntd.0002143

115. Monel B, Compton AA, Bruel T, et al. Zika virus induces massive cytoplasmic vacuolization and paraptosis-like death in infected cells. Embo J. 2017. doi:10.15252/embj.201695597

116. Johnson RC, Nackers F, Glynn JR, et al. Association of HIV infection and Mycobacterium ulcerans disease in Benin. Aids. 2008;22(7):901-903. 
http://www.ncbi.nlm.nih.gov/entrez/query.fcgi?cmd=Retrieve\&db=PubMed\&dopt=Ci tation\&list_uids=18427211.

117. Christinet V, Comte E, Ciaffi L, et al. Impact of human immunodeficiency virus on the severity of Buruli ulcer disease: Results of a retrospective study in cameroon. Open Forum Infect Dis. 2014. doi:10.1093/ofid/ofu021

118. Vincent QB, Ardant MF, Marsollier L, Chauty A, Alcais A, Franco-Beninese Buruli Research G. HIV infection and Buruli ulcer in Africa. Lancet Infect Dis. 2014;14(9):796797. doi:10.1016/S1473-3099(14)70882-5

119. O'Brien DP, Comte E, Serafini M, et al. The urgent need for clinical, diagnostic, and operational research for management of Buruli ulcer in Africa. Lancet Infect Dis. 2014;14(5):435-440. doi:10.1016/S1473-3099(13)70201-9

120. Lowe E, Fan AR, Jiang J, et al. Blocking Protein Secretion with Novel Small Molecule Inhibitors of Sec61 Represents a Potential Treatment Strategy Against Hematologic Malignancies. Blood. 2019. doi:10.1182/blood-2019-123782

121. Demangel C, High S. Sec61 blockade by mycolactone: a central mechanism in Buruli ulcer disease. Biol Cell. 2018. doi:10.1111/boc.201800030 


\section{Figure legends}

Figure 1: Current understanding of mycolactone's distribution in infected hosts

The mycolactone structure shown corresponds to $M$. ulcerans-derived stereoisomers $A / B$, the red line indicating the region where $A$ and $B$ differ ${ }^{47}$.

Figure 2: Immune functions known, or suspected to be suppressed by mycolactone.

Figure created in Biorender.com.

Figure 3: Mycolactone inhibits Sec61-dependent protein translocation.

Most secreted and membrane proteins are delivered to the ER, as ribosome bound nascent chains. Their transfer to the Sec61 complex starts with binding of a hydrophobic signal peptide contained in their sequence (highlighted in yellow) to a signal recognition particle (SRP, Stage 1 ), which then binds to its cognate receptor (SR) located in the ER membrane (Stage 2). Mycolactone inhibits the subsequent recognition of nascent chain by Sec61 (Stage 3), thus preventing its engagement in the translocon (Stage 4) and insertion in the ER membrane (Stage 5). Sec61 substrates that fail to translocate in the ER are directed to the proteasome for cytosolic degradation.

Figure 4: Proposed mechanism for mycolactone-mediated cytotoxic activity.

Mycolactone-mediated Sec61 blockade causes the cytosolic accumulation of mycolactonesusceptible Sec61 substrates blocked in translocation, which are unable to fold properly outside the oxidizing environment of the ER and without membrane insertion. This results in upregulation of Hsp70 (the stress-induced form of the Hsc70 molecular chaperone critical for nascent protein folding) and Hsp90 (which forms with Hsp70 a multichaperone machinery regulating proteostasis). Sec61 substrates that resist mycolactone inhibition of Sec61 (such as Type III transmembrane proteins) do not fold properly in the ER due to the lack of mycolactone-sensitive molecular chaperones like BIP. This triggers the UPR, reflected by Xbp1 mRNA splicing by the ER-resident stress sensor IRE1 $\alpha$. ISR/UPR sensor PERK phosphorylates elF2 $\alpha$, which stimulates the translation of ATF4 transcription factor. Activated ATF4 then induces the transcriptional upregulation of the pro-apoptotic factor $\mathrm{CHOP}$. 
Figure 1

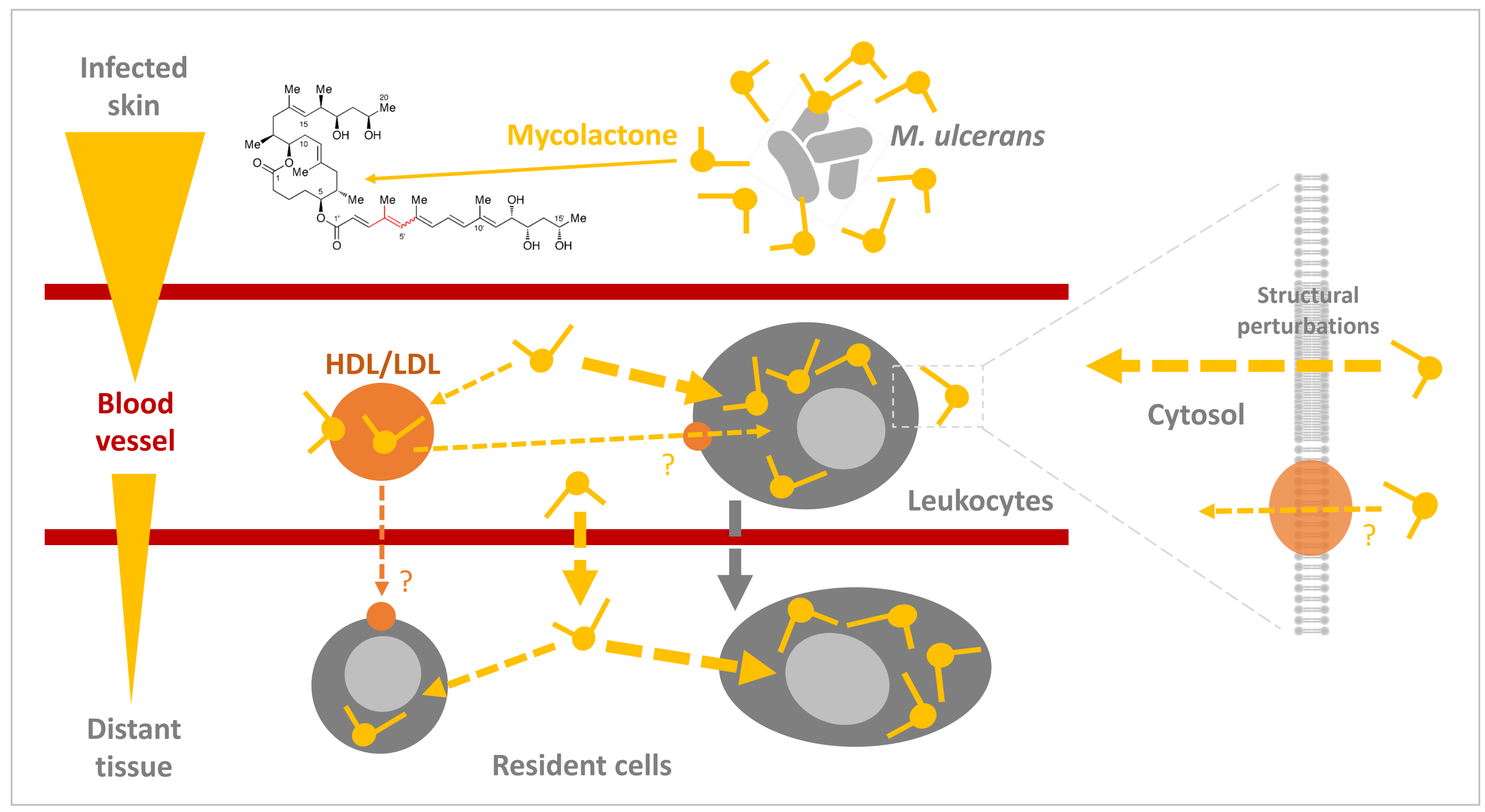


Figure 2

T cell priming by dendritic cells

Dendritic cells

- Maturation, migration

- Antigen presentation

- Cytokine / Chemokine

production

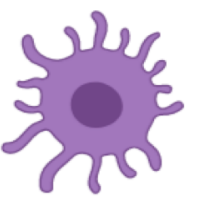

Macrophages

- IFN- $\gamma$ signaling

- Cytokine production

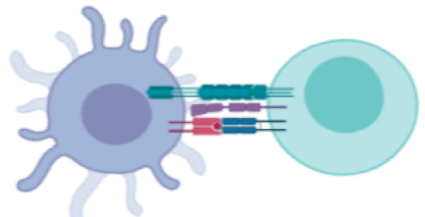

T

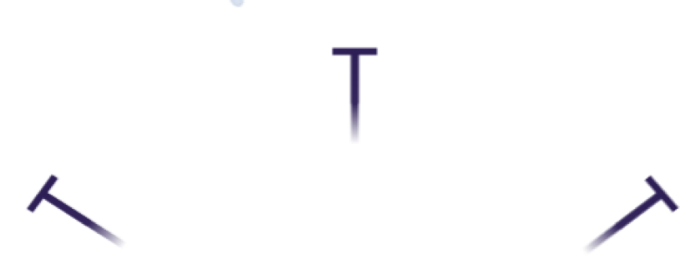

Mycolactone
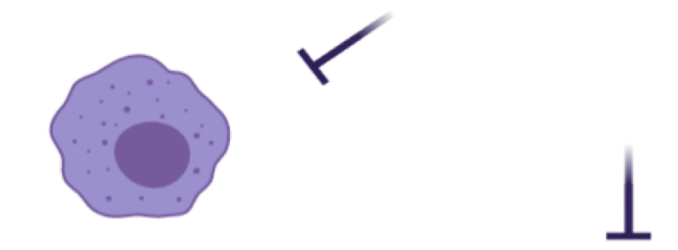

Neutrophils

TNF- $\alpha$ production

Inflammation sensing and migration?

\section{T cells}

(2) Homing

- Response to TCR stimulation

- Cytokine/Chemokine production

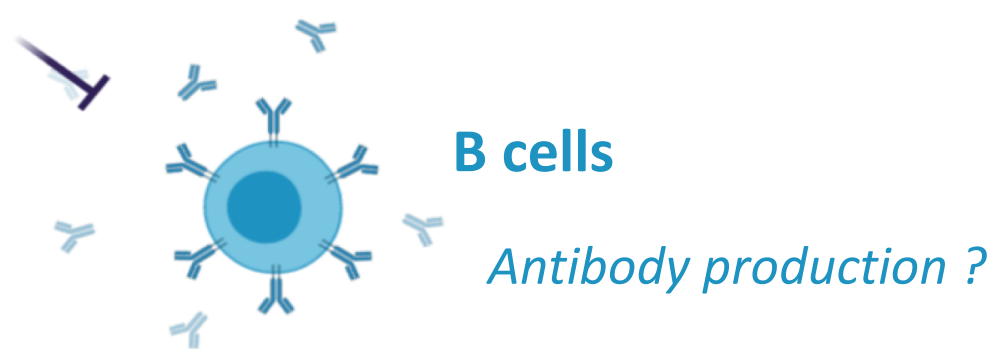


Figure 3

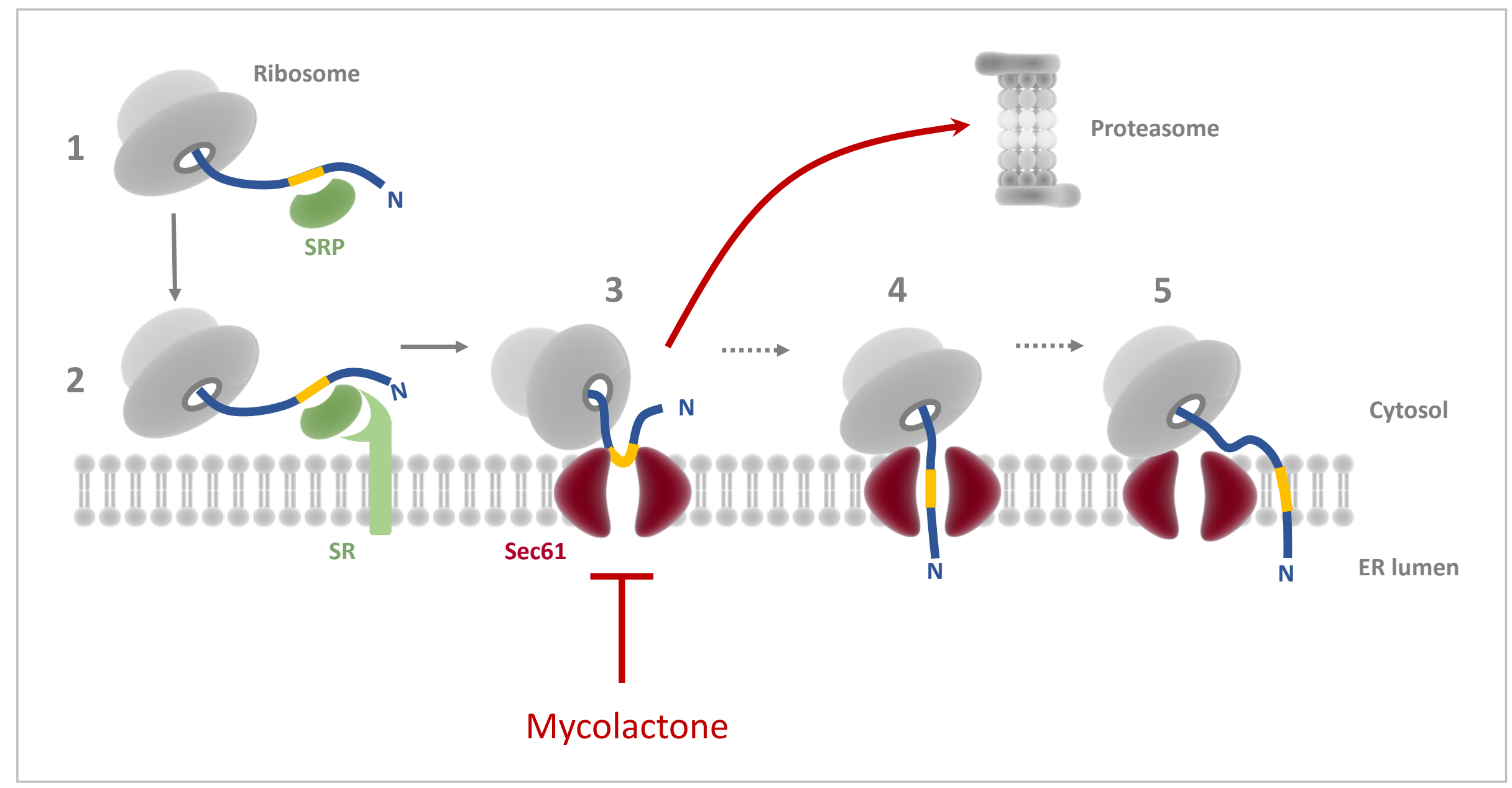


Figure 4

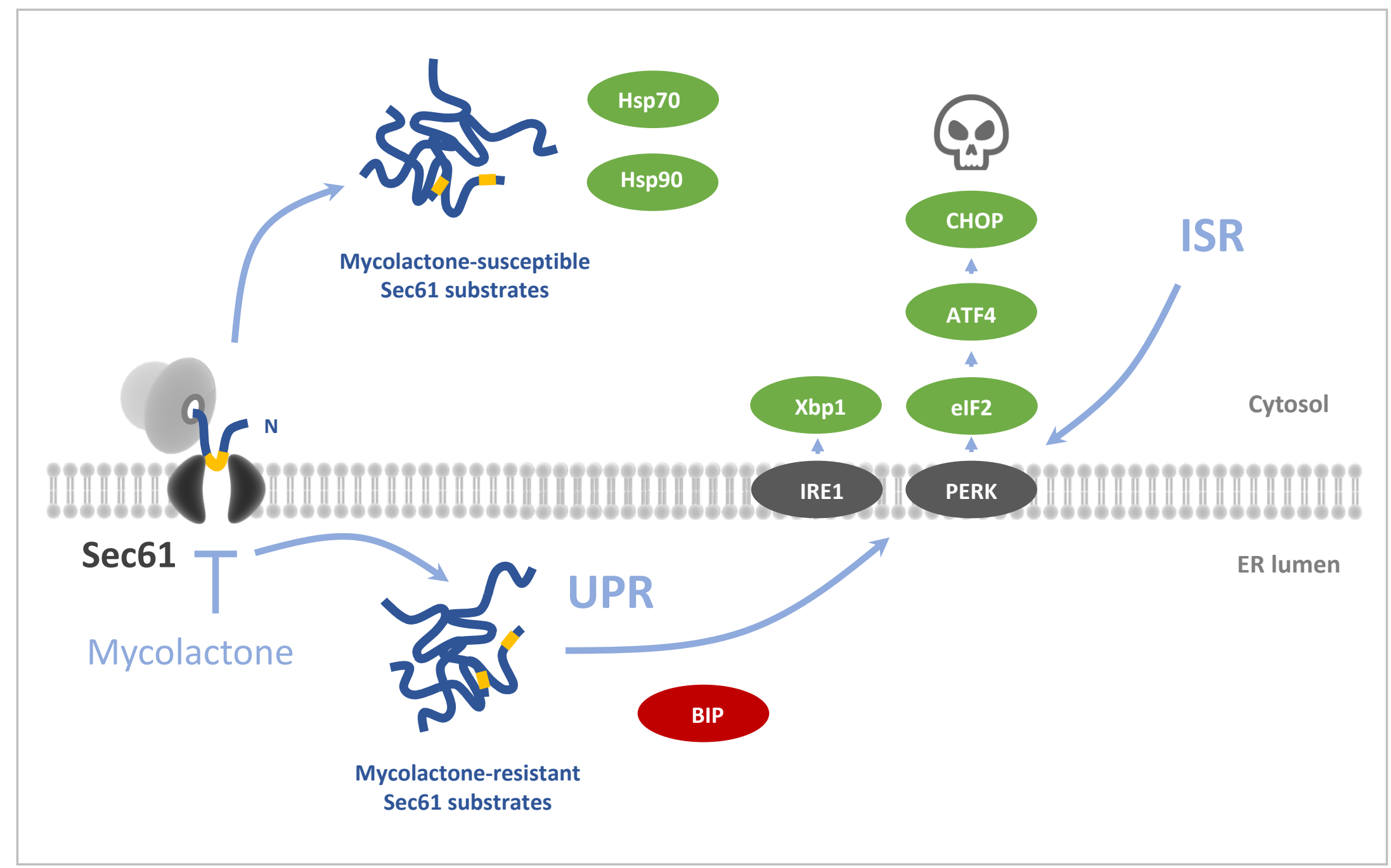

